\title{
Assaying mechanosensation *
}

\author{
Martin Chalfie ${ }^{1 \S}$, Anne C. Hart ${ }^{2 \S}$, Catharine H. Rankin ${ }^{3 \S}$, and Miriam B. \\ Goodman $^{4 \mathcal{S}}$ \\ ${ }^{1}$ Department of Biological Sciences, Columbia University, New York NY, USA \\ ${ }^{2}$ Department of Neuroscience, Brown University, Providence RI, USA \\ ${ }^{3}$ University of British Columbia, Vancouver, BC, Canada \\ ${ }^{4}$ Department of Psychology, Stanford University, Stanford CA, USA
}

\section{Table of Contents}

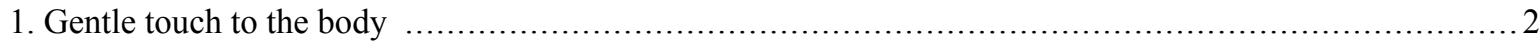

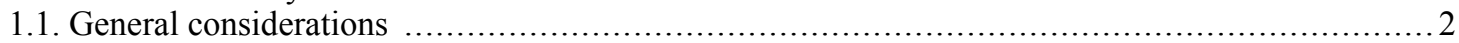

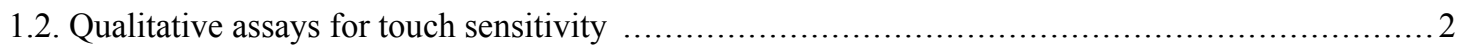

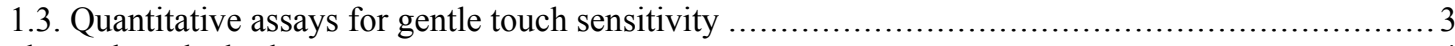

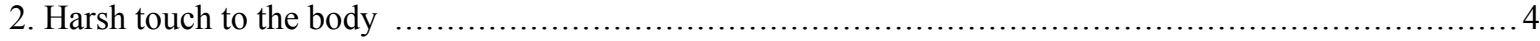

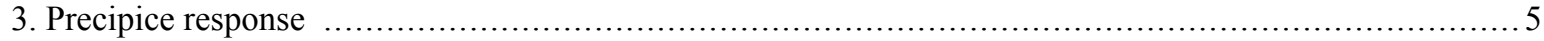

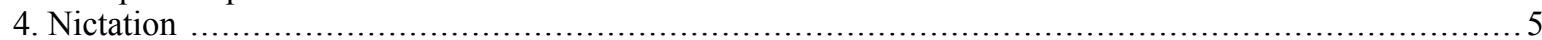

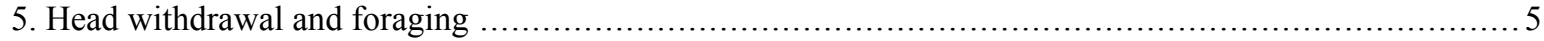

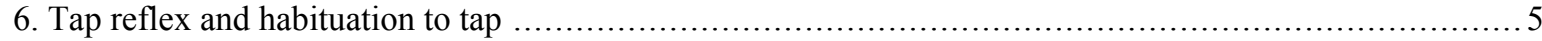

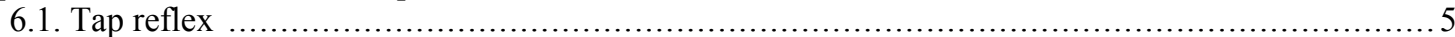

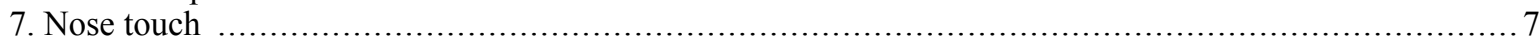

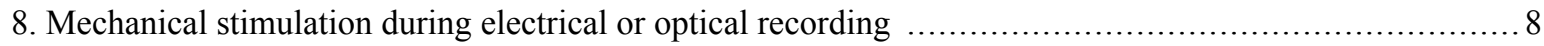

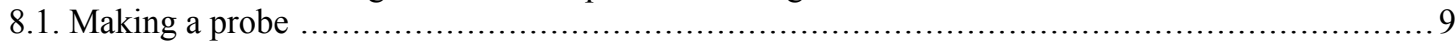

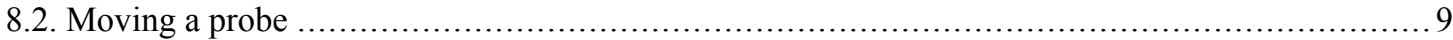

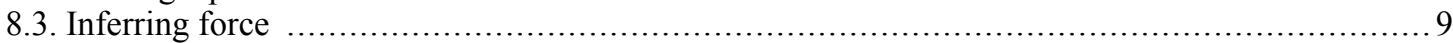

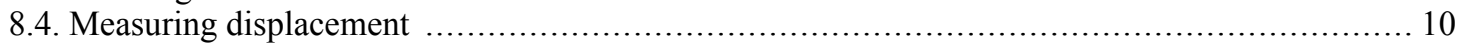

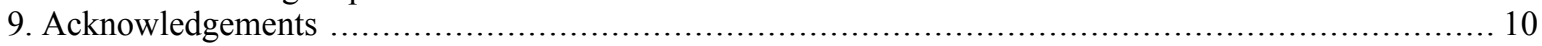

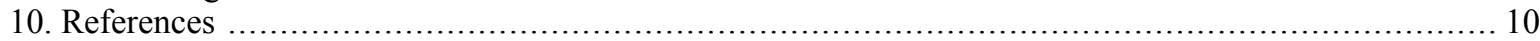

*Edited by: Oliver Hobert Last revised: July 1, 2013. Published July 31, 2014. This chapter should be cited as: Chalfie, M., et al. Assaying mechanosensation (July 31, 2014), WormBook, ed. The C. elegans Research Community, WormBook, doi/10.1895/wormbook.1.172.1,

http://www.wormbook.org.

Copyright: (C) 2014 Martin Chalfie, Anne C. Hart, Catharine H. Rankin and Miriam B. Goodman. This is an open-access article distributed under the terms of the Creative Commons Attribution License, which permits unrestricted use, distribution, and reproduction in any medium, provided the original author and source are credited.

§ To whom correspondence should be addressed: mc21@columbia.edu, hart@brown.edu, crankin@psych.ubc.ca, or mbgoodman@stanford.edu 


\begin{abstract}
C. elegans detect and respond to diverse mechanical stimuli using neuronal circuitry that has been defined by decades of work by $C$. elegans researchers. In this WormMethods chapter, we review and comment on the techniques currently used to assess mechanosensory response. This methods review is intended both as an introduction for those new to the field and a convenient compendium for the expert. A brief discussion of commonly used mechanosensory assays is provided, along with a discussion of the neural circuits involved, consideration of critical protocol details, and references to the primary literature.
\end{abstract}

\title{
1. Gentle touch to the body
}

\subsection{General considerations}

C. elegans senses a variety of mechanical stimuli. These stimuli include gentle touch stimulus delivered to the body (Chalfie and Sulston, 1981; Sulston et al., 1975), harsh touch to the midbody (Way and Chalfie, 1989), harsh touch to the head or tail (Chalfie and Wolinsky, 1990), nose touch (Kaplan and Horvitz, 1993), and texture (Sawin et al., 2000). Gentle touch to the body is sensed by six sensory neurons (ALML/R, PLML/R, AVM, and PVM). An important feature of any assay for gentle touch mediated by these cells is that it not be confused with the response to other mechanical signals. Thus, care should be taken to touch the animals in a way that will not stimulate other mechanosensory neurons. In practice, one should avoid too harsh a stimulus and not touch the animals too near the tip of the head or tail.

\subsection{Qualitative assays for touch sensitivity}

\subsubsection{Stroking with an eyebrow hair}

The initial and most generally used method to test for gentle touch sensitivity is to stroke the animals with an eyebrow hair that has been glued to the end of a toothpick (Chalfie and Sulston, 1981) (Figure 1). Eyebrow hairs are used because they are usually not cut and are, thus, finely tapered. They also can be obtained with a minimum of discomfort. Some papers stated that the hairs are from eyelashes. However, removing eyelashes is a much more painful process. Various glues can be used to secure the hairs, but the hair should be placed so that its shaft extends straight from the end of the toothpick and does not curve away from the tip.

The hair is sterilized by dipping it into a 70\% ethanol solution and dried by shaking (don't flame sterilize the hair). Sometimes bacteria accumulate on the hair; they can be removed by poking the hair into the agar on a spare plate.

Animals are touched by stroking the hair across the body just behind the pharynx (for the anterior touch response) or just before the anus (for the posterior touch response; see Figure 1). Actually touching the animals in any position along the touch receptor processes will generate a response. Touching the animals near the middle of the body (near the vulva in adult hermaphrodites), however, yields ambiguous results because both the anterior and posterior touch circuits can be activated. The animals should not be poked with the end of the hair, since this provides a stronger stimulus and can sometimes evoke a response, even in touch-insensitive animals. Similarly, animals should not be touched with the end of a platinum worm pick. Animals should not be touched at either the tip of the nose or the tip of the tail as even animals lacking the six touch sensing cells often respond.

The touch assay is really a differential assay, since animals that are dead or paralyzed will also not respond to the stroking. Routinely, animals are said to be touch-sensitive if they respond to stroking with the eyebrow hair by stopping movement toward the hair or move away from the hair (sometimes the touch stops moving animals without having them reverse their direction of movement). Touch-insensitive (Mec) animals fail to respond to the hair, but do respond to prodding with a worm pick. A partial response is one in which the animals react to only some of the touches.

Touch sensitivity mediated by the anterior touch cells can be assayed in severely uncoordinated animals by monitoring pharyngeal pumping (Chalfie et al., 1985; M. Chalfie, unpublished data). Touching animals in the anterior slows the rate of pharyngeal pumping. Another output of the touch circuitry is the defecation rhythm, which is reset by stroking the anterior of the animal with the eyebrow hair and is dependent on genes needed for the function of the touch receptor (mec-4, mec-5, and mec-9; Thomas, 1990). 
A

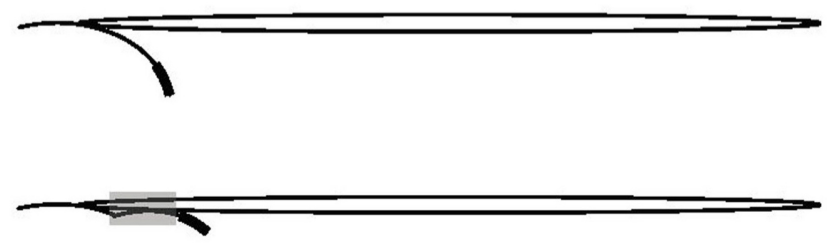

B

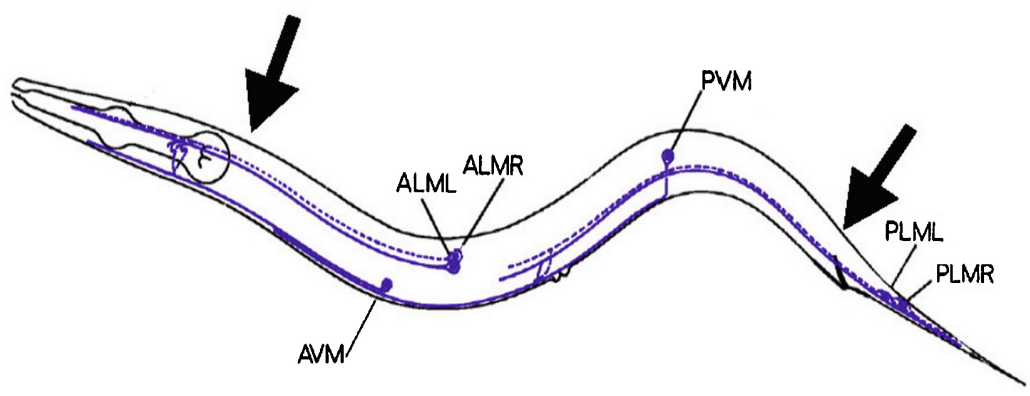

Figure 1. Using an eyebrow hair to test gentle touch sensitivity. (A) Positioning (top panel) and gluing (bottom panel) the eyebrow hair to the tooth pick. The thickened black line indicates the shaft of the hair; the gray area indicates the location of the glue. (B) Animals should be touched by stroking the hair across the body at the positions of the arrows. The six touch receptor neurons are indicated.

\subsubsection{Tapping the plate}

Wild-type animals will move (adults usually reverse direction) in response to their plate being tapped (Chalfie and Sulston, 1981). This stimulus often happens when plates are placed on the stage of a dissecting microscope. Touch-insensitive animals do not respond to this tapping. Although not as accurate a measure of touch sensitivity as touching with an eyebrow hair, this is a rapid assay that has been used to screen for touch-insensitive mutants (Chalfie and $\mathrm{Au}, 1989$ ). Plates with $\mathrm{F} 2$ progeny of mutagenized parents were dropped from about $1-2 \mathrm{~cm}$ above the stage of the microscope and then examined for animals that did not move. Candidate touch mutants were then tested with the eyebrow hair and worm pick to determine if they were touch insensitive. Catharine Rankin and coworkers designed an electric tapper to deliver taps to plates at defined intervals for their studies examining the touch circuitry and habituation of the touch response (Rankin, 1991; Wicks and Rankin, 1995).

\subsection{Quantitative assays for gentle touch sensitivity}

The above assays treat touch sensitivity as an all-or-none phenomenon. But, even touch-insensitive animals sometimes respond to the first touch. Two methods below provide a more quantitative measure of the touch response: counting the responses to multiple touches, and touching with defined stimuli.

\subsubsection{Scoring the result of multiple touches}

The first instance of using multiple touches with an eyebrow hair to measure the degree of insensitivity to touch was by Hobert et al. (1999). In this assay animals are touched ten times (alternating head and tail touches) and a score is given for the number of positive responses. At least thirty animals are examined and mean percentage score is obtained. Since individual touches are not always the same, the values obtained in this assay are not truly quantitative. Nonetheless, by using multiple animals, relatively subtle difference in touch sensitivity can be revealed (e.g., Hobert et al., 1999; Zhang and Chalfie, 2002). In addition, by separately scoring head and tail responses, differences between the two can also be uncovered (Zhang et al., 2004; Chen and Chalfie, 2014). In using this method one should be careful to distinguish between animals that habituate more rapidly than wild type from those that respond less frequently to the touch stimulus. The animals with the former defect should show a response pattern in which the animals respond less frequently to successive touches; animals with the latter defect should show no pattern to the failures. The experimenter should also determine whether the animals are responding equally (in terms of number of responses) to touches in the head or tail. This method can be further modified by gently placing the hair next to the animals instead of stroking the hair. This modification identifies subtler diffderences in the touch response (Chen and Chalfie, 2014). 


\subsubsection{Using micro von Frey hairs}

von Frey hairs have been used clinically for over a century to test human touch sensitivity. They consist of a series of flexible fibers that are touched end-on to skin and bend once a specific force has been applied. The longer and thinner the fiber, the less force is required to bend it. These calibrated fibers are then used to determine the forces needed to provoke a touch sensation when placed on the skin. We adapted this method to C. elegans by using fine monofilament nylon sutures (7-0 and smaller; Iris Chin, M. Goodman, and M. Chalfie, unpublished data). A short length of suture (Sharpoint ${ }^{\mathrm{TM}}$, Surgical Specialties Corporation) is attached perpendicularly to the end of a glass capillary $(100 \mu \mathrm{L}$ volume) with epoxy and calibrated using an analytical balance. The force (in $\mu$ newtons $(\mu \mathrm{N})$ ) required for bending is calculated as the weight (in $\mathrm{mg}$ ) found for the bent fiber X $9.8 \mathrm{~m} / \mathrm{s}^{2}$. A limitation of this method is that fibers that deliver less than $10 \mu \mathrm{N}$ of force are difficult to use and calibrate because they bend with the air currents normally found in the lab.

Typically, we mount the capillary on a manual micromanipulator with the fiber perpendicular to the agar surface, position a worm underneath the fiber, and make contact by moving the $\mathrm{z}$-axis of the micromanipulator. Animals are touched in the same locations as described above for testing body touch with eyebrow hairs, and the response is noted. Ninety percent of wild-type hermaphrodites respond to a $10 \mu \mathrm{N}$ of force; virtually all animals respond to forces $>100 \mu \mathrm{N}$. In contrast, hermaphrodites homozygous for mec-3 and mec- 6 null mutations fail to respond to a $100 \mu \mathrm{N}$ force. Approximately $10 \%$ of mec-4 (u253) animals respond to a $100 \mu \mathrm{N}$ force (the difference may reflect some activity from the PVD cells, which are defective in mec-3 animals).

\subsubsection{Force- and displacement-clamp systems for mechanical stimulation}

Whether using human hairs or nylon fibers, classical methods for delivering mechanical stimulation provide limited control over stimulus strength. New devices based on micro-electrical mechanical systems (MEMS) techniques and soft-lithography for designing and fabricating silicon devices provide such control and have been developed to measure body mechanics and touch sensation in worms (Park et al., 2007; Doll et al., 2009; Park et al., 2011; Petzold et al., 2011; Ghanbari et al., 2012; Petzold et al., 2013).

An integrated system for analysis of gentle body touch consists of a force-sensing cantilever integrated into a feedback circuit enabling experimenters to control the size and duration of mechanical stimulation. With this system, either displacement or force can be controlled. Stimuli are delivered from above to animals crawling on an agar surface and the system also includes a digital camera for recording behavioral output. With this system, Petzold et al. (2013) showed that wild-type worms can detect sub- $\mu \mathrm{N}$ forces and sub- $\mu \mathrm{m}$ indentations.

\section{Harsh touch to the body}

Harsh touch to the body was originally measured by prodding animals with a platinum wire in the midsection of the body (Chalfie and Sulston, 1981; Way and Chalfie, 1989). Nonmoving gravid adults are prodded at or just posterior to the vulva. Animals respond by initiating locomotion, usually by backing up. Animals to be assayed should be grown in the continuous presence of food. Animals who are starved and animals that have passed through the dauer stage often fail to respond to harsh touch regardless of the functional status of the PVD sensory neurons (Way and Chalfie, 1989).

Harsh touch is assessed in animals in which the function of the ALM and PLM neurons have been perturbed as these neurons mediate response to both gentle and harsh touch. Response to harsh touch is mediated by the PVD sensory neurons and perhaps the FLP sensory neurons (Way and Chalfie, 1989; Chatzigeorgiou et al., 2010b). The phenotype is called "Touch abnormal" (abbreviated Tab) to distinguish it from "Mechanosensory defective" (abbreviated $\mathrm{Mec}$ ) animals, i.e., animals that cannot respond to gentle touch.

Subsequent research on harsh touch has refined the methodology. Li et al. (2011), used a platinum pick with a $20 \mu \mathrm{m}$ thick and $30 \mu \mathrm{m}$ long flattened tip. Force is applied from above animals by pressing down with the edge of the pick. Five trials are delivered in this manner with a 2-10 minute inter-trial interval. These authors estimated the force delivered by recording similar pressure on an analytical balance, recording the change in mass produced by stimulation, and converting this to force according to $F=\Delta m a$, where $a$ is the gravitational force $\left(9.8 \mathrm{~m} / \mathrm{s}^{2}\right)$ and $\Delta m$ is the change in mass. Forces on the order of $100 \mu \mathrm{N}$ are generated by this method. 


\section{Precipice response}

Although the animals are reported to avoid the edge of an agar chunk, little is known about this behavior.

\section{Nictation}

C. elegans dauers can be observed atop mold filaments moving in an undulating fashion independent of plate orientation. (Croll and Matthews, 1977) An overtly similar behavior called nictation likely plays a role in the dispersal of other nematode dauers including infectious species or Pristionchus pacificus (Brown et al., 2011). C. elegans dauer nictation can be assessed by placing dauers on fields of $25 \mu \mathrm{m}$ tall agar posts with a radius and a spacing of $25 \mu \mathrm{m}$ (Lee et al., 2011), or by laying cotton gauze on the agar surface. Nictation requires functional IL2 neurons and is required for $C$. elegans dispersal in laboratory assays using Drosophila as carriers.

\section{Head withdrawal and foraging}

The nose of $C$. elegans generally moves in a rhythmic dorsal/ventral pattern as animals feed on a bacterial lawn on the surface of agar plates. In this context, rhythmic nose swinging is called foraging. Aberrant foraging rates and patterns of movement can be observed in animals with defective OLQ and IL1 labial neurons or in animals with defects in RMD motorneurons, which are synaptic targets of these labial neurons (Hart et al., 1995).

Animals respond to touch to the side of the nose by rapidly moving their nose away from the stimulus; this is called head withdrawal. Response is mediated by the OLQ and IL1 labial sensory neurons. Genes required for head withdrawal include glr-1 and trpa-1, but not mec-4 or osm-9 (Hart et al., 1995; Kindt et al., 2007b). A hair, similar in size to the hair used in the nose touch assay, is placed on the agar plate parallel to the direction of forward locomotion, so that the side of the animal's nose will just touch the hair at the maximal extent of the foraging nose swings. Animals respond to contact by rapidly moving their nose away from the hair or initiating a reversal. Practice is required to learn where to lay down the hair and to discriminate between the rapid withdrawal motion versus normal foraging motion. Foraging and head withdrawal assays should be undertaken by observers blind to genotype or treatment. Thin bacterial lawns for these assays can be prepared by releasing a single drop of an overnight OP50 culture over a standard NGM plate (see Maintenance of $C$. elegans, Section 3). Allow the liquid to soak into the plate. The plate should be used within a few hours or should be sealed with Parafilm ${ }^{\circledR}$ and stored at $4^{\circ} \mathrm{C}$ for weeks. Allow stored plates to return to room temperature before use.

\section{Tap reflex and habituation to tap}

\subsection{Tap reflex}

The tap-withdrawal protocol measures the responses of a worm to a single tap or a series of successive taps (trains of taps), given to the side of a standard NGM agar plate. The taps/trains cause the worm's environment to vibrate briefly, and this causes the adult worm to swim backwards for some distance (termed a reversal). This technique produces a quantifiable measure of both the frequency and the magnitude of this reversal response. The tap is a complex stimulus as it activates both anterior and posterior body-touch cells, as well as PVD (Wicks and Rankin, 1995). The head cell activation promotes backward movement while the tail cell activation promotes forward movement and so the response to tap is an integration of the activation of opposing subcircuits (Wicks and Rankin, 1995). 
The response to tap can be studied using video recording and manual scoring, or by automated tracker (see WormBook chapter Keeping track of worm trackers). For both types of assays worms are placed on an NGM agar filled plate and are recorded either by a high-resolution camera lens alone, or through the lens of a dissecting microscope. A mechanical tapper is arranged to hit the center of one side of the plate. In the original, manually scored, experiments the tapper was composed of an electromagnetic relay run by a stimulator (Rankin et al., 1990) that delivered a force of approximately 1 to 2 newtons $(\mathrm{N})$ per tap. In the tracker based experiments, a variety of tappers have been used including a Mindstorm robot (i.e., Kindt et al., 2007a) and a solenoid mounted so that the plunger strikes the Petri plate (i.e., Swierczek et al., 2011). Regardless of the source of the tap the reversal response to tap can be affected by several variables:

1. The intensity of the tap. The tap response is graded and stronger stimuli produce larger reversals (Chiba and Rankin, 1990; Timbers et al., 2013).

2. The age of the worm. Chiba and Rankin (1990), showed that as worms got larger so too did reversals, while Timbers et al. (2013) showed that younger worms reacted differently to stimuli of different intensities and older worms were less sensitive to changes in intensity. Timbers et al. (2013) measured tap intensity using Laser Doppler vibrometry (LDV) (OFV-5000, Polytec Inc.), which measures the velocity (m/s) or displacement (m) of a moving surface by detecting the Doppler shift of a reflected laser beam.

3. The agar in the plates. As agar plates age they dry out and lead to differences in the ease with which worms can move. This can be controlled for by only using plates within a certain age range, or by weighing plates prior to use.

4. The ambient temperature and humidity in the room where the testing is done. This is probably the result of changes in the agar and ease of movement; it is an important variable to control. At the very least, experimental and control animals must always be run together, on the same day, at about the same time, under all of the same conditions. By taking note of temperature and humidity conditions for each animal it will be possible to determine whether variations in either variable affected the outcome of the experiment.

5. Other sources of stimulation. It is important to leave the lids on the plates during testing: the stimulus of removing the lid changes the worms behavior, and can lead to problems as the agar dries our.

6. Experience. The tap response decrements with previous exposure to taps (Giles and Rankin, 2009). In addition, mechanosensory experience in general can affect the response to tap. For example, if plates are kept in an incubator with a vibrating fan, or if plates are handled a lot prior to testing, worms will respond to tap differently than worms without that experience.

Once an experiment has been run and recorded, the behavior must be scored. This can be done by hand or by using software with one of the tracker programs (see Keeping track of worm trackers). For manual scoring, each animal's movement is scored by tracing movement from the video image onto a transparent acetate sheet taped to the video monitor, going frame by frame through the video. The adult worm will usually ( $90 \%$ to $95 \%$ of the time) respond to the tap with a reversal response, with the size and number of the responses decreasing as stimuli are repeated through the habituation run. In a typical reversal response, a stopped or forward-moving animal moves backwards for a distance (usually less than 1 or 2 worm lengths) and then either remains still or re-initiates forward movement in a new direction. To score the response, a person notes the pre-tap position of the worm, and then traces the total distance the worm reversed (i.e., track length) onto the acetate sheet. Because the head of the worm often makes small side-to side foraging movements, reversals are most accurately scored by tracing the path of the tail. These "scored" tracings are then scanned into a computer and the length of each is measured using NIH Image. The values from this program, representing the reversal/acceleration magnitude, can then be directly transported into a statistical package for data analysis. Alternatively, response to tap can be quantified using more recently developed automated systems that combine stimulus delivery with image capture and analysis (e.g., Swierczek et al., 2011).

With any of these analyses it is important to define the parameters for identifying and scoring a reversal. A scoring manual should be developed with rules for scoring variations from this behavior (i.e., the reversal must occur within 1 second of the tap, or if the worm is already swimming backwards when the tap is delivered this is a "missing data point", etc.). In some cases, an animal will respond with acceleration forward rather than a reversal. In a typical acceleration response, a stopped or forward-moving worm either initiates forward movement or increases its speed, respectively. Generally, in experiments with adult wild-type worms, these acceleration responses are 
simply scored as missing data points (an adult worm will accelerate to tap only about $5 \%$ of the time). Under other circumstances or protocols, such as with larval, mutant, or laser-ablated animals, the acceleration response occurs more often and is therefore scored. If a worm accelerates after the tap, the acceleration is scored by measuring either the distance the worm moves or the speed the animal moves during the $1 \mathrm{sec}$ interval before the tap and subtracting it from the distance/speed the worm moves during the $1 \mathrm{sec}$ interval after the tap. If the measurement for the sec after the tap is $\geq 1.75 \mathrm{X}$ the measurement for the sec before the tap, the response is considered an acceleration. It is important to note that the reversal response and the acceleration response are two qualitatively different outcomes that cannot easily be compared. The frequency of reversals and accelerations are also important measures of the behavior of the worm, and are scored simply by counting the number of instances of either response. Reversal magnitude and reversal probability appear to be independent measures and can be affected differently by a given mutation (Kindt et al., 2007a).

Prior to undertaking tap reflex experiments, it would be advisable to also read further about the tap reflex (Giles and Rankin, 2009).

\section{Nose touch}

C. elegans responds to gentle touch to the nose by initiating backward locomotion. (Kaplan and Horvitz, 1993) This response is the "Nose touch response" or Not assay. Response to nose touch is mediated by the ASH, OLQ and FLP sensory neurons. The two polymodal ASH sensory neurons mediated $60 \%$ of the wild type response to nose touch. Their ciliated, sensory endings are located at the tip of the nose where they detect numerous noxious stimuli, often in combination with other neurons. ASH neurons play a role in response to light touch to the nose, high osmolarity (Hart et al., 1999), low pH (Sambongi et al., 1999), heavy metal ions (Bargmann et al., 1990; Sambongi et al., 1999), UV light (Ward et al., 2008), volatile repellent chemicals (Troemel, 1999), quinine, and detergents (Hilliard et al., 2004).

Nose touch is assayed by laying a hair on the surface of the plate in front of the animal. As an animal moves forward, it contacts the hair with the tip of the nose perpendicular to the direction of movement. Some practice is required to anticipate where the hair must be laid down for an animal to run into it at 90 degrees. Normal animals immediately initiate backward locomotion. Defective animals either hurdle over the hair or slide their nose along the hair. An individual animal is tested no more than 10 times in a row to avoid inducing habituation. You cannot induce lethargic animals to move or reverse direction by touching them as this diminishes response to nose touch, but you can raise the assay plate $1 \mathrm{~cm}$ and let it drop onto the stage. Nose touch can be assayed either on very thin bacterial lawns or off food. Response on food is more robust and is preferred. Animals that are moved to thin lawns recover within $10 \mathrm{~min}$ and respond in roughly $90 \%$ of trials. Response by animals who have wandered off the bacterial lawn is slightly diminished: animals respond in roughly $80 \%$ of trials. Response is dramatically reduced in animals that have been recently moved by the investigator to a plate lacking food: animals respond in roughly $30 \%$ of trials (Chao et al., 2004). The hair should be wiped off every few trials to eliminate bacteria and water that may adhere. Normal animals will ignore a wet or slimy hair. A folded Kimwipe type wiper or clean bare fingers can be used. In the latter case, avoid using lotions, creams, or other surfactants. Don't flame the hair. Individual hairs can be reused used for weeks.

The hair used in the Not assay must be of an appropriate size. Too thick will result in all animals responding, too thin will cause even animals of mutant genotype to respond. Hair thickness has definite variation between individuals; arm hair from a female researcher or an eyelash from a male researcher would be a good place to start. Fine paintbrush hairs can also be used. The root of a hair is taped to the small end of a glass Pasteur pipette or to a thin wooden stick. Slightly thicker hairs are required for nose touch assays on food. Generally the very tip of a hair is too thin and the animal must impact a thicker (and less distal) part of the hair in the nose touch trial. Thin bacterial lawns for nose touch assays can be prepared by releasing a single drop of an overnight OP50 culture over a standard NGM plate. Allow the liquid to soak into the plate. To prevent bacterial growth and too much thickening of the bacterial lawn, the plate should be used within a few hours or should be sealed with Parafilm and stored at $4^{\circ} \mathrm{C}$ for weeks. Allow stored plates to return to room temperature before use. If the bacterial lawn is too thick, the animals may crawl through the food over or under the hair and avoid the nose touch stimulus.

Each hair is tested by comparing the response of N2 and glr-1 animals. (Hart et al., 1995). N2 animals should respond robustly; glr- 1 animals should fail in $90 \%$ of trials. (Note that eat-4 animals are so defective in their response to nose touch that they are not useful as a control (Lee et al., 1999; Raizen and Avery, 1994)). At a bare minimum at least 5 animals should be tested at 10 trials each on two different days along with controls. Response is 
expressed as fractional or \% response. C. elegans adapt to nose touch after a rapid series of repeated trials. The nose touch trials must be given in fairly rapid succession to adapt the animals' response (without accidentally hitting the animal over the head with the hair). To measure habituation, compare the $\%$ response in the first 10 trials to the $\%$ response in the trials 40 through 50 .

\section{Mechanical stimulation during electrical or optical recording}

Controlled mechanical stimuli have been delivered to worms during electrophysiological recordings or optical imaging. Mechanical stimuli can be defined as a function applied displacement (Suzuki et al., 2003; Kindt et al., 2007a; Kang et al., 2010; Li et al., 2011; Chen and Chalfie, 2014) or force (O'Hagan et al., 2005; Bounoutas et al., 2009; Arnadóttir et al., 2011; Geffeney et al., 2011). The choice between the two measures has been largely a matter of technical convenience, since it was unknown whether $C$. elegans senses the indentation or the force applied by the stimulator probe. When delivering force pulses, the size of the probe also affects the stimulus achieved, since the force applied via a small probe generates a larger stress (pressure) than the same force applied by a larger probe.

A study by Petzold et al. (2013) used a custom-designed and fabricated (micro-electrical mechanical) MEMS-based device to show that behavioral responses evoked by stimulating the receptive field of the touch receptor neurons follow indentation more closely than force. This new finding suggests that controlling displacement and measuring body indentation will be the method of choice going forward.

Electrical and optical recordings of $C$. elegans mechanoreceptor neurons conducted to date are summarized in Tables 1 and 2, respectively.

Table 1. Mechanical stimulation during electrical recording

\begin{tabular}{|l|l|l|l|l|}
\hline Neuron & Stimulus & $\begin{array}{l}\text { Amplitude } \\
(\mathbf{m i n}-\mathbf{m a x})\end{array}$ & Probe diameter & Citation \\
\hline PLM & Force & $0.05-5 \mu \mathrm{N}$ & $10-20 \mu \mathrm{m}$ & $\begin{array}{l}\text { O'Hagan et al. 2005; } \\
\text { Bounoutas et al., } \\
\text { 2009; Arnadóttir et } \\
\text { al., 2011 }\end{array}$ \\
\hline PLM & & & Kang et al. 2010 \\
\hline CEP & Displacement & ns & ns & Kang et al. 2010 \\
\hline PDE & Displacement & $0.5-4 \mu \mathrm{m}$ & ns & Li et al., 2011 \\
\hline PVD & Displacement & $10 \mu \mathrm{m}$ & ns & Li et al., 2011 \\
\hline ASH & Displacement & $20 \mu \mathrm{m}$ & ns & Geffeney et al., 2011 \\
\hline ns $=$ not specified & Force & $5-80 \mu \mathrm{N}$ & $20 \mu \mathrm{m}$ & \\
\hline
\end{tabular}

Table 2. Mechanical stimulation during calcium imaging

\begin{tabular}{|l|l|l|l|l|l|}
\hline Neuron & Stimulus & Amplitude (max) & Probe diameter & Indicator & Citation \\
\hline ALM & Displacement & $10 \mu \mathrm{m}$ & $\begin{array}{l}15 \mu \mathrm{m}, \text { glass and } \\
\text { platinum }\end{array}$ & $\begin{array}{l}\text { Cameleon } \\
\text { YC2.12 and } \\
\text { YC2.13 }\end{array}$ & $\begin{array}{l}\text { Suzuki et al., } \\
2003\end{array}$ \\
\hline ASH & Displacement & $\mathrm{ns}$ & $50 \mu \mathrm{m}$, glass & $\begin{array}{l}\text { Cameleon } \\
\text { YC2.12* }\end{array}$ & $\begin{array}{l}\text { Hilliard et al., } \\
2005\end{array}$ \\
\hline ASH & Displacement & $8 \mu \mathrm{m}$ & $10 \mu \mathrm{m}$, glass & $\begin{array}{l}\text { Cameleon } \\
\text { YC2.12* }\end{array}$ & $\begin{array}{l}\text { Kindt et al., } \\
2007 \mathrm{~b}\end{array}$ \\
\hline OLQ & Displacement & $8 \mu \mathrm{m}$ & $15 \mu \mathrm{m}$, glass & Cameleon YCD3 & $\begin{array}{l}\text { Kindt et al., } \\
2007 \mathrm{~b}\end{array}$ \\
\hline ALM, PLM & Displacement & & $15 \mu \mathrm{m}$, glass & $\begin{array}{l}\text { Cameleon } \\
\text { YC3.12 }\end{array}$ & $\begin{array}{l}\text { Kindt et al., } \\
2007 \mathrm{a}\end{array}$ \\
\hline
\end{tabular}




\begin{tabular}{|l|l|l|l|l|l|}
\hline Neuron & Stimulus & Amplitude (max) & Probe diameter & Indicator & Citation \\
\hline CEP & Displacement & & $10 \mu \mathrm{m}$, glass & Cameleon YC2.1 & Kindt et al, 2007a \\
\hline ALM & Displacement & $10 \mu \mathrm{m}$ & $\mathrm{ns}$ & $\begin{array}{l}\text { Cameleon, } \\
\text { YC2.12 }\end{array}$ & $\begin{array}{l}\text { Chatzigeorgiou et } \\
\text { al., 2010a }\end{array}$ \\
\hline ALM & Displacement & $\begin{array}{l}\text { 'harsh': } 30-50 \mu \mathrm{m} \\
\text { at } 2.8 \mu \mathrm{m} / \mathrm{s}\end{array}$ & 'sharp' glass & $\begin{array}{l}\text { Cameleon, } \\
\text { YC3.12 }\end{array}$ & $\begin{array}{l}\text { Chatzigeorgiou et } \\
\text { al., 2010b }\end{array}$ \\
\hline ALM & Displacement & 'gentle': $10 \mu \mathrm{m}$ & $10 \mu \mathrm{m}$ & $\begin{array}{l}\text { Cameleon, } \\
\text { YC3.12 }\end{array}$ & $\begin{array}{l}\text { Chatzigeorgiou et } \\
\text { al., 2010b }\end{array}$ \\
\hline ALM & Displacement & $6 \mu \mathrm{m}$ & $22 \mu \mathrm{m}$ & GCaMP3 & $\begin{array}{l}\text { Chen and Chalfie, } \\
\text { 2014 }\end{array}$ \\
\hline PVD & Displacement & $\begin{array}{l}\text { 'harsh': } 30-50 \mu \mathrm{m} \\
\text { at } 2.8 \mu \mathrm{m} / \mathrm{s}\end{array}$ & 'sharp' glass & Cameleon, YC2.3 & $\begin{array}{l}\text { Chatzigeorgiou et } \\
\text { al., 2010b }\end{array}$ \\
\hline FLP & Displacement & Nose touch: $8 \mu \mathrm{m}$ & $\mathrm{ns}$ & $\begin{array}{l}\text { Cameleon, } \\
\text { YC2.3† }\end{array}$ & $\begin{array}{l}\text { Chatzigeorgiou et } \\
\text { al., 2011 }\end{array}$ \\
\hline FLP & Displacement & Body touch: $2 \mu \mathrm{m}$ & ns & Cameleon, YC2.3 & $\begin{array}{l}\text { Chatzigeorgiou et } \\
\text { al., 2011 }\end{array}$ \\
\hline
\end{tabular}

\subsection{Making a probe}

By contrast with the fibers used for classical behavioral assays, probes suitable for stimulation during electrophysiological or optical recordings are made from glass rods or capillaries. In a few instances, platinum wires have also been used. This is because worms are immobilized and immersed in physiological saline during cellular recordings and glass is stiff enough to move smoothly through aqueous solutions. To make a glass probe, use a pipette puller (e.g., Sutter P-97 or similar) to pull a glass rod (OD $1.0 \mathrm{~mm}$ or $1.5 \mathrm{~mm}$ ) to a fine tip (ca. $1-2 \mu \mathrm{m}$ in diameter). Next, use a microforge to fire polish the tip to create a large ball at the end of the rod. To deliver forces reliably, the probe must be softer than the worm. Conversely, to faithfully indent the worm, the probe should be at least as stiff as the worm. Wild type (N2) worms have a body stiffness of approximately $\sim 1 \mathrm{~N} / \mathrm{m}$ (Park et al., 2007; Park et al., 2011; Petzold et al., 2011; Petzold et al., 2013). (Note that stiffness depends on cuticle composition and muscle tone, since manipulations that alter these factors are known to both increase and decrease body stiffness (Park et al., 2007; Petzold et al., 2011; Petzold et al., 2013)).

\subsection{Moving a probe}

Several techniques have been reported in the literature that enable experimenters to control the timing and intensity of mechanical stimulation during electrical or optical recordings (Suzuki et al., 2003; O'Hagan et al., 2005; Kang et al. 2010; Chatzigeorgiou et al., 2010a; Chatzigeorgiou et al., 2010b; Chatzigeorgiou et al., 2011; Geffeney et al., 2011). They share the use of piezoelectric devices or motorized manipulators to generate controlled movements. A report by Hao et al. (2013) of piezoelectrical strategies for indenting cultured mammalian sensory neurons provides a useful overview of what is possible with today's technology.

\subsection{Inferring force}

We measure force in units of newtons, but force is not measured directly. Whether the probe is crafted from nylon, glass or silicon, we infer force from a model and a measurement of another quantity related to force according to the model.

The model behind the calibration procedure for von Frey hairs is called 'Euler Buckling' and, roughly speaking, it says that pushing a flexible fiber against a harder object causes the fiber to buckle, applying a force that depends on the length and thickness of the fiber. Once buckling has occurred, no additional force is delivered to the object. This is the principle behind calibrating a von Frey fiber by pressing it against the pan of an analytical balance, which measures mass. But mass still is not force and another model, namely the relationship between mass and the gravitational force, $F=m \mathrm{a}$ is needed to compute force. Note that the geometry of the procedure matters, the von Frey hair must be pushed against the object at a right angle and not brushed along its surface. The latter situation is ruled by a different and much more complex model. 
Hooke's law, $F=-\mathrm{k} x$, is the model that describes how glass probes have been used to deliver force to worms (O'Hagan et al., 2005; Geffeney et al., 2011). In this model, the probe is like a spring attached to a solid surface and force, $F$, is inferred from, $x$, the displacement of the probe and its spring constant, $k$. So, in order to infer $F$, we need to measure $x$ during each stimulation and determine $k$ for each probe. The next section discusses procedures for making these measurements.

\subsubsection{Calibrating probes: strategies for measuring the spring constant}

Generally speaking, we apply a known force and measure the resulting displacement and use Hooke's law relating force to displacement to compute a spring constant, $\mathrm{k}$. The simplest method for applying a known force is gravity: hang a known mass from the tip of the probe $(F=m \mathrm{a}$, where a is the gravitational constant $)$ and measure the resulting displacement. The known mass can be made from a short piece of metal wire (copper or gold) and the gravity-induced displacement can be measured by mounting the probe on a horizontal microscope such as the one integrated into certain microforges (e.g., Narishige). Once these measures are in place, the spring constant, $\mathrm{k}$, is computed according to:

$$
k_{\text {ref }}=(m \cdot a) / d
$$

Where, $m$ is the wire's mass, a is the gravitational constant, and $d$ is the displacement produced by the mass.

A probe intended for experiments can be calibrated directly or indirectly, the choice depends on the range of spring constant needed to deliver the desired forces. The gentle touch receptor neurons detect forces between $10^{-8}$ and $10^{-6} \mathrm{~N}$ (O'Hagan et al., 2005), while the ASH nociceptor detects forces between $10^{-6}$ and $10^{-4} \mathrm{~N}(\mathrm{Geffeney}$ et al., 2011). An indirect method was used for probes crafted to stimulate the TRNs. Briefly, O'Hagan et al. (2005) made a stiffer (higher spring constant) reference probe. The spring constant for the reference probe was calculated from Hooke's law after measuring the displacement produced by a known force applied by hanging a small mass (a length of fine copper or gold wire) from the end of the probe:

The reference probe then provides a known force and is used to bend the stimulation probe while measuring the resulting displacement. This measurement has an error of at least $10 \%$, which means that forces inferred from probe displacement cannot be more accurate than this.

Geffeney et al. (2011) used an alternative indirect method in which the reference probe was a microelectromechanical systems (MEMS)-based force-sensor. The principle is the same, but, because the MEMS-based probe measures displacement directly and is calibrated with more precision, this alternative method is more accurate and precise.

\subsection{Measuring displacement}

A simple method for measuring force probe displacement during electrical or optical recording is to collect videos of probe movement in free solution, $d_{\text {free }}$, and while being pressed against the side or nose of a worm, $d_{\text {stim }}$. Next, measure $d_{\text {free }}$ and $d_{\text {stim }}$ from the videos. O'Hagan et al. (2005) did this 'by hand' using ImageJ, while Geffeney et al. (2011) used Visible ${ }^{\mathrm{TM}}$ motion detection software (Reify Corporation) to make these measurements. The difference between $d_{\text {free }}$ and $d_{\text {stim }}$ gives the distance that the probe was bent during contact with the worm. Next, calculate force from Hooke's Law: $F=\left(d_{\text {free }}-d_{\text {stim }}\right) k_{\text {stim }}$.

\section{Acknowledgements}

MC was funded by NIH grant GM30997. MBG is funded by NIH grants NS047715 (MBG) and EB006745 (MBG and B. L. Pruitt). ACH is funded by NS055813. CHR is funded by an NSERC Discovery grant.

\section{References}

Arnadóttir, J., O'Hagan, R,. Chen, Y., Goodman, M.B., and Chalfie, M. (2011). The DEG/ENaC protein MEC-10 regulates the transduction channel complex in Caenorhabditis elegans touch receptor neurons. J. Neurosci. 31, 12695-12704. Abstract Article 
Bargmann, C.I., Thomas, J.H., and Horvitz, H.R. (1990). Chemosensory cell function in the behavior and development of Caenorhabditis elegans. Cold Spring Harb. Symp. Quant. Biol. 55, 529-538. Abstract Article

Bounoutas, A., Hagan, R.O., and Chalfie, M. (2009). The multipurpose 15-protofilament microtubules in C. elegans have specific roles in mechanosensation. Curr. Biol. 19, 1362-1367 Abstract Article

Brown, F.D., D'Anna, I., Sommer, R.J. (2011). Host-finding behaviour in the nematode Pristionchus pacificus. Proc. Biol. Sci. 278, 3260-3269. Abstract Article

Chalfie, M., and Au, M. (1989). Genetic control of differentiation of the C. elegans touch receptor neurons. Science 243, 1027-1033. Abstract Article

Chalfie, M., and Sulston, J. (1981). Developmental genetics of the mechanosensory neurons of Caenorhabditis elegans. Dev. Biol. 82, 358-370. Abstract Article

Chalfie, M., and Wolinsky, E. (1990). The identification and suppression of inherited neurodegeneration in Caenorhabditis elegans. Nature 345, 410-416. Abstract Article

Chalfie, M., Sulston, J.E., White, J.G., Southgate, E., Thomson, J.N., and Brenner, S. (1985). The neural circuit for touch sensitivity in C. elegans. J. Neurosci. 5, 956-964. Abstract

Chatzigeorgiou, M., Grundy, L., Kindt, K.S., Lee, W.H., Driscoll, M., and Schafer, W.R. (2010a). Spatial asymmetry in the mechanosensory phenotypes of the C. elegans DEG/ENaC gene mec-10. J. Neurophys. 104, 3334-3344. Abstract Article

Chatzigeorgiou, M., Yoo, S., Watson, J.D., Lee, W.H., Spencer, W.C., Kindt, K.S., and Schafer, W.R. (2010b). Specific roles for DEG/ENaC and TRP channels in touch and thermosensation in C. elegans nociceptors. Nat. Neurosci. 13, 861-868. Abstract Article

Chatzigeorgiou, M., and Schafer, W.R. (2011). Lateral facilitation between primary mechanosensory neurons controls nose touch perception in C. elegans. Neuron 70, 299-309. Abstract Article

Chao, M.Y., Komatsu, H., Fukuto, H.S., Dionne, H.M., and Hart, A.C. (2004). Feeding status and serotonin rapidly and reversibly modulate a Caenorhabditis elegans chemosensory circuit. Proc. Natl. Acad. Sci. U. S. A. 101, 15512-15517. Abstract Article

Chen, X., and Chalfie, M. (2014). Modulation of C. elegans touch sensitivity is integrated at many levels. J. Neurosci. 34, 6522-6536. Abstract Article

Chiba, C.M., and Rankin, C.H. (1990). A developmental analysis of spontaneous and reflexive reversals in the nematode Caenorhabditis elegans. J. Neurobiol. 21, 543-554. Abstract Article

Croll, N.A. (1975). Components and patterns in the behavior of the nematode Caenorhabditis elegans. J. Zool. 176, 159-176. Article

Croll, N.A., and Matthews, B.E. (1977). Biology of Nematodes (New York: John Wiley \& Sons). Abstract

Doll, J.C., Harjee, N., Klejwa, N., Kwon, R., Coulthard, S.M., Petzold, B., Goodman, M.B., and Pruitt, B.L. (2009) SU-8 force sensing pillar arrays for biological measurements. Lab Chip 9, 1449-1454. Abstract Article

Geffeney, S.L, Cueva, J.G., Glauser, D.A., Doll, J.C., Lee, T.H., Montoya, M., Karania, S., Garakani, A.M., Pruitt, B.L., and Goodman, M.B. (2011). DEG/ENaC but not TRP channels are the major mechanoelectrical transduction channels in a C. elegans nociceptor. Neuron 71, 845-857. Abstract Article

Ghanbari, A., Nock, V., Johari, S., Blaikie, R., Chen, X., and Wang, W. (2012). A micropillar-based on-chip system for continuous force measurement of $C$. elegans J. Micromech. Microeng. 22095009 doi:10.1088/0960-1317/22/9/095009 Article

Giles, A.C., and Rankin, C.H. (2009). Behavioral and genetic characterization of habituation using Caenorhabditis elegans. Neurobiol. Learn. Mem. 92, 139-146. Abstract Article 
Hart, A.C., Kass, J., Shapiro, J.E., and Kaplan, J.M. (1999). Distinct signaling pathways mediate touch and osmosensory responses in a polymodal sensory neuron. J. Neurosci. 19, 1952-1958. Abstract

Hart, A.C., Sims, S., and Kaplan, J.M. (1995). Synaptic code for sensory modalities revealed by C. elegans GLR-1 glutamate receptor. Nature 378, 82-85. Abstract Article

Hao, J., Ruel, J., Coste, B., Roudaut, Y., Crest, M., and Delmas, P. (2013). Piezo-electrically driven mechanical stimulation of sensory neurons. Methods Mol. Biol. 998, 159-170. Abstract Article

Hilliard, M.A., Bergamasco, C., Arbucci, S., Plasterk, R.H., and Bazzicalupo, P. (2004). Worms taste bitter: ASH neurons, QUI-1, GPA-3 and ODR-3 mediate quinine avoidance in Caenorhabditis elegans. EMBO J. 23, 1101-1111. Abstract Article

Hobert, O., Moerman, D.G., Clark, K.A., Beckerle, M.C., and Ruvkun, G. (1999). A conserved LIM protein that affects muscular adherens junction integrity and mechanosensory function in Caenorhabditis elegans. J. Cell Biol. 144, 45-57. Abstract Article

Husson, S.J., Steuer Costa, W., Schmitt, C., and Gottschalk, A. Keeping track of worm trackers (September 10, 2012), WormBook, ed. The C. elegans Research Community, WormBook, doi/10.1895/wormbook.1.156.1, http://www.wormbook.org.

Kang, L., Gao, J., Schafer, W.R., Xie, Z., and Xu, X.Z. (2010). C. elegans TRP family protein TRP-4 is a pore-forming subunit of a native mechanotransduction channel. Neuron 67, 381-391. Abstract Article

Kaplan, J.M., and Horvitz, H.R. (1993). A dual mechanosensory and chemosensory neuron in C. elegans. Proc. Natl. Acad. Sci. U. S. A. 90, 2227-2231. Abstract Article

Kindt, K.S., Quast, K.B., Giles, A.C., Hendrey, D., Nicastro, I,. Rankin, C.H. and Schafer, W.R. (2007a). Dopamine mediates food dependent modulation of sensory plasticity in C. elegans. Neuron 55, 662-676. Abstract Article

Kindt, K.S., Viswanath, V., Macpherson, L., Quast, K., Hu, H., Patapoutian, A., and Schafer, W.R. (2007b). Caenorhabditis elegans TRPA-1 functions in mechanosensation. Nat. Neurosci. 10, 568-577. Abstract Article

Lee, H., Choi, M.K., Lee, D., Kim, H.S., Hwang H., Kim, H., Park, S., Paik, Y.K., and Lee, J. (2011). Nictation, a dispersal behavior of the nematode Caenorhabditis elegans, is regulated by IL2 neurons. Nat. Neurosci. 15, 107-112. Abstract Article

Lee, R.Y., Sawin, E.R., Chalfie, M., Horvitz, H.R., and Avery, L. (1999). EAT-4, a homolog of a mammalian sodium-dependent inorganic phosphate cotransporter, is necessary for glutamatergic neurotransmission in Caenorhabditis elegans. J. Neurosci. 19, 159-167. Abstract

Li, W., Kang, L., Piggott, B.J., Feng, Z., and Xu, X.Z. (2011). The neural circuits and sensory channels mediating harsh touch sensation in Caenorhabditis elegans. Nat. Commun. 2, 315. Abstract Article

O'Hagan, R., Chalfie, M., and Goodman, M.B. (2005) The MEC-4 DEG/ENaC channel of Caenorhabditis elegans touch receptor neurons transduces mechanical signals. Nat. Neurosci. 8, 43-50. Abstract Article

Park, S.J., Goodman, M.B., and Pruitt, B.L. (2007). Analysis of nematode mechanics by piezoresistive displacement clamp. Proc. Natl. Acad. Sci. U. S. A. 104, 17376-17381. Abstract Article

Park, S.J., Petzold, B.C., Goodman, M.B., and Pruitt, B.L. (2011). Piezoresistive cantilever force-clamp system. Rev. Sci. Instrum. 82, 043703. Abstract Article

Petzold, B.C., Park, S.J., Mazzochette, E.A., Goodman, M.B., and Pruitt, B.L. (2013). MEMS-based force-clamp analysis of the role of body stiffness in C. elegans touch sensation. Integr. Biol. (Camb) 5, 853-864. Abstract Article

Petzold, B.C., Park, S.J., Ponce, P., Roozeboom, C., Powell, C., Goodman, M.B., and Pruitt, B.L. (2011). Caenorhabditis elegans body mechanics are regulated by body wall muscle tone. Biophys. J. 100, 1977-1985. Abstract Article 
Raizen, D.M., and Avery, L. (1994). Electrical activity and behavior in the pharynx of Caenorhabditis elegans. Neuron 12, 483-495. Abstract Article

Rankin, C.H. (1991). Interactions between two antagonistic reflexes in the nematode Caenorhabditis elegans. J. Comp. Physiol. [A] 169, 59-67. Abstract Article

Rankin, C.H., Beck, C.D., and Chiba, C.M. (1990). Caenorhabditis elegans: a new model system for the study of learning and memory. Behav. Brain Res. 37, 89-92. Abstract Article

Sambongi, Y., Nagae, T., Liu, Y., Yoshimizu, T., Takeda, K., Wada, Y., and Futai, M. (1999). Sensing of cadmium and copper ions by externally exposed ADL, ASE, and ASH neurons elicits avoidance response in Caenorhabditis elegans. Neuroreport 10, 753-757. Abstract Article

Sanyal, S., Wintle, R.F., Kindt, K.S., Nuttley, W.M., Arvan, R., Fitzmaurice, P., Bigras, E., Merz, D.C., Hébert, T.E., and Van Der Kooy, D. (2004). Dopamine modulates the plasticity of mechanosensory responses in Caenorhabditis elegans. EMBO J. 23, 473-482. Article

Sawin, E.R., Ranganathan, R., and Horvitz, H.R. (2000). C. elegans locomotory rate is modulated by the environment through a dopaminergic pathway and by experience through a serotonergic pathway. Neuron 26 , 619-631. Abstract Article

Stiernagle, T. Maintenance of C. elegans (February 11, 2006), WormBook, ed. The C. elegans Research Community, WormBook, doi/10.1895/wormbook.1.101.1, http://www.wormbook.org. Article

Sulston, J., Dew, M., and Brenner, S. (1975). Dopaminergic neurons in the nematode Caenorhabditis elegans. J. Comp. Neurol. 163, 215-226. Abstract Article

Suzuki, H., Kerr, R., Bianchi, L., Frøkjaer-Jensen, C., Slone, D., Xue, J., Gerstbrein, B., Driscoll, M., and Schafer, W.R. (2003). In vivo imaging of $C$. elegans mechanosensory neurons demonstrates a specific role for the MEC-4 channel in the process of gentle touch sensation. Neuron 39, 1005-1017. Abstract Article

Swierczek, N., Giles, A., Rankin C.H., and Kerr, R., (2011). High-throughout behavioral analysis in C. elegans. Nat. Methods 8, 592-598. Abstract Article

Thomas, J.H. (1990). Genetic analysis of defecation in Caenorhabditis elegans. Genetics 124, 855-872. Abstract

Timbers, T.A., Giles, A.C., Ardiel, E.A., Kerr, R.A., and Rankin, C.H. (2013). Intensity discrimination deficits cause habituation changes in middle-aged Caenorhabditis elegans, Neurobiol. Aging 34, 621-631. Abstract Article

Troemel, E.R. (1999). Chemosensory signaling in C. elegans. Bioessays 21, 1011-1020. Abstract Article

Ward, A., Liu, J., Feng, Z., and Xu, XZ. (2008). Light-sensitive neurons and channels mediate phototaxis in $C$. elegans. Nat. Neurosci. 11, 916-922. Abstract Article

Way, J.C., and Chalfie, M. (1989). The mec-3 gene of Caenorhabditis elegans requires its own product for maintained expression and is expressed in three neuronal cell types. Genes Dev. 3, 1823-1833. Abstract Article

Wicks, S.R., and Rankin, C.H. (1995). Integration of mechanosensory stimuli in Caenorhabditis elegans. J. Neurosci. 15, 2434-2444. Abstract

Zhang, S., Arnadottir, J., Keller, C., Caldwell, G.A., Yao, C.A., and Chalfie, M. (2004). MEC-2 is recruited to the putative mechanosensory complex in $C$. elegans touch receptor neurons through its stomatin-like domain. Curr. Biol. 14, 1888-1896. Abstract Article

Zhang, Y., and Chalfie, M. (2002). MTD-1, a touch-cell-specific membrane protein with a subtle effect on touch sensitivity. Mech. Dev. 119, 3-7. Abstract Article

All WormBook content, except where otherwise noted, is licensed under a Creative

Commons Attribution License. 